\title{
The diagnosis of pulmonary embolism
}

\author{
S. TALBOT \\ M.B., B.S., M.R.C.P. \\ P. D. GRIFFITHS \\ B.SC., PH.D.
}

Nottingham General Hospital, Nottingham

\section{Summary}

A blind assessment of abnormalities of pulmonary scintiscans in patients with pulmonary emboli and other clinical conditions showed that there was no abnormality specific for pulmonary embolism. However, a normal lung scan virtually excluded pulmonary embolism and an area, or areas, of absent perfusion was confirmatory evidence of embolism, without infarction, if the chest radiograph was normal, and of embolism with infarction if the clinical and radiographic findings were compatible.

A controlled analysis of biochemical and electrocardiographic abnormalities associated with pulmonary embolism showed that abnormalities of liver function and blood urea were more frequent than in a group of patients suspected of, but not having, emboli. A similar analysis of the electrocardiograms showed that a Q3R3S1 with $T$ wave inversion over the right ventricular leads and lead III ( \pm avf) that developed or regressed was pathognomonic of embolism, but other features were of little value.

It is suggested that all hospital patients should have an electrocardiogram performed on admission so that serial changes may be assessed and that lung scanning should be used as a screening test in patients suspected of having pulmonary embolism.

\section{Introduction}

Pulmonary embolism is a frequent cause of death (Short, 1952) and may be frequent in clinical practice (Carlotti et al., 1947). The diagnosis of this condition in life is difficult because of the non-specific clinical features and the absence of a diagnostic test. Many tests have been reported to show specific abnormalities in patients arbitrarily classified as having pulmonary emboli but, since a control group of patients must be considered in any evaluation, these results are of doubtful significance.

This study attempts to compare the abnormalities of the scintiscan and biochemical tests and of the electrocardiogram in patients with pulmonary emboli and controls with similar clinical features to see if any test is specific and if any is a useful screening test. In view of the difficulties of clinical diagnosis a large number of autopsies were investigated.
Study

\section{Pulmonary scintiscanning}

From 1969 to 1971 lung scans were performed on 213 patients following injection of 1-2 mc of 99MTc-HSA macroaggregates $(20-80 \mu$ in diameter). A single 3 in.-headed scanner was used which presented the results as colour scans. In all but fortythree cases posterior as well as anterior scans were obtained. The scans were assessed by a physicist without the clinical opinion being known as:

(1) normal;

(2) non-specific abnormality: an area or areas of the lung(s) with diminished perfusion;

(3) severely abnormal-an area or areas of the lung(s) with absent perfusion [comparison of radioactive counts was made with the mediastinal (or background) count].

The patients were scanned for suspected pulmonary emboli or assessment of pulmonary perfusion. Often the diagnosis was subsequently revised. The clinical or radiographic diagnosis of pulmonary embolism or infarction (with presumed embolism) was:

(1) a definite pulmonary infarction if there was a venous thrombosis as judged by swelling ( $>0.5 \mathrm{in}$ ) and tenderness of a limb together with elevation of the diaphragm and radiographic shadowing on the side corresponding to pleuritic pain and signs of consolidation. Dyspnoea but not haemoptysis was essential;

(2) a probable pulmonary infarction if there was a venous thrombosis (as defined) together with pleuritic pain, dyspnoea and haemoptysis. The plain radiographic features were not specific for pulmonary infarction. There were also five patients with clinical and radiographic evidence of pulmonary infarction but no signs of venous thrombosis;

(3) a definite pulmonary embolism if it was detected on angiography or autopsy.

\section{Results}

Analysis of the scans of 174 patients is presented in Table 1. Of the thirty-nine cases excluded before the analysis, twenty-three had severe cardiac failure (in which embolism is common) and thirteen were scanned more than 2 weeks after their 'embolic episode'. 
TABLE 1. Scintiscan abnormalities in pulmonary embolism/infarction

\begin{tabular}{lcccr}
\hline & \multicolumn{3}{c}{ Type of scintiscan } \\
\cline { 2 - 4 } Clinical diagnosis & Normal & $\begin{array}{c}\text { Non-specific } \\
\text { abnormality of } \\
\text { perfusion }\end{array}$ & $\begin{array}{c}\text { Area(s) } \\
\text { of absent } \\
\text { perfusion }\end{array}$ & $\begin{array}{c}\text { Total } \\
\text { patients }\end{array}$ \\
\hline Probable pulmonary infarction & 1 & 12 & 1 & 14 \\
Definite pulmonary infarction & 0 & 18 & 5 & 23 \\
Definite pulmonary embolism & 0 & 6 & 2 & 8 \\
Other cardio-respiratory & 4 & 71 & 11 & 86 \\
$\quad$ conditions & 0 & 4 & 12 & 16 \\
Pulmonary neoplasia & 19 & 8 & 0 & 27 \\
Normal & 24 & 119 & 31 & 174 \\
Total & & & & \\
\hline
\end{tabular}

There was a significant difference in the incidence of any type of abnormal scan between patients with emboli and normal patients $\left(\chi^{2}=35.7, P<0.01\right)$. However, the difference in the incidence of nonspecific abnormal scans $\left(\chi^{2}=5.45, P<0.02\right)$ was significant in cases with emboli/infarction compared with all the patients with other conditions. Complete absence of perfusion in one lung was found, principally in cases of carcinoma (seven cases) and emphysema (nine cases), and was not found with pulmonary embolism. However, discrete areas of absent perfusion in one or both lungs of lobar or sub-lobar distribution were found in eight embolic cases out of forty-five compared with only two patients with other non-neoplastic conditions. It is conditions such as chest infections, left ventricular failure and obstructive airways disease that may simulate pulmonary embolism. The difference in incidence of this scintiscan appearance was significant for pulmonary embolism/infarction $\left(\chi^{2}=11 \cdot 4, P<0.01\right)$. Neoplasia produced a similar abnormality in five cases.

\section{Discussion}

Previous studies have described abnormalities of the scan in cases of pulmonary embolism (Haynie, Hendrick and Schreiber, 1963). Such cases may or may not show angiographic abnormalities but the lung scan is almost always abnormal if there is an abnormal angiogram and the investigations are performed within $24 \mathrm{hr}$ of each other (Moser et al., 1966). The clinical criteria for the diagnosis of pulmonary embolism are often not specified, but this study with rigid clinical and radiographic criteria confirmed the value of the lung scan as a screening test to exclude pulmonary embolism.

Angiograms were performed in four patients considered to have emboli and all were normal. In retrospect the normal chest radiograph and lung scan would have excluded embolism in these patients. Also in fifteen patients with radiographic abnormalities, and in six cases suspected of having emboli but with normal radiographs, normal lung scans helped to exclude pulmonary emboli.

The lung scan is abnormal in cardiac failure (James et al., 1971), emphysema (Beerel et al., 1969) and asthma (Wagner, 1968). There was no specific abnormality due to embolism although an area of absent uptake was confirmatory evidence of a clinical and radiographic diagnosis.

In fifty-four patients the portable method of scanning of Maclean et al. (1967) was used and thirteen of these patients had normal scans and were therefore spared further investigation and treatment. This takes $20 \mathrm{~min}$ and could be utilized in many provincial hospitals to exclude embolism.

\section{Biochemical investigations}

In view of the reports of the diagnostic value of biochemical investigations (Wacker and Snodgras, 1960), a retrospective analysis of serum enzyme tests, the blood urea, and serum bilirubin was undertaken of patients autopsied between 1966 and 1970. Case notes of patients dying of thrombo-embolism and of a control group of patients suspected of having emboli or infarction in life, which were excluded at autopsy, were selected if details of the above tests were recorded. Only investigations taken after pulmonary embolism had occurred were included and therefore most cases had evidence of infarction.

\section{Results}

The percentage of abnormal results for each test is presented in Table 2 and this includes the normal range for each test. Seventy-four embolic cases and ninety-two control cases were included.

The LDH and the SGOT were not of diagnostic value, but abnormalities of the serum bilirubin, the SGPT and the alkaline phosphate were more frequent in the embolic group. These were often associated with increased urinary urobilinogen. The abnormal levels of these tests were also greater than those of the control group. 
TABLE 2. Figures in parentheses indicate the total number of tests performed

\begin{tabular}{ccc}
\hline $\begin{array}{c}\text { Biochemical } \\
\text { investigation }\end{array}$ & $\begin{array}{c}\text { Pulmonary } \\
\text { embolism } \\
\text { percentage }\end{array}$ & Control \\
\hline $\begin{array}{c}\text { Raised LDM } \\
(>365 \text { i.u. })\end{array}$ & $25 \%(28)$ & $29 \%(52)$ \\
$\begin{array}{c}\text { Raised SGOT } \\
(>24.5 \text { i.u. })\end{array}$ & $20 \%(24)$ & $12 \%(48)$ \\
$\begin{array}{c}\text { Raised SGPT } \\
(>15 \text { i.u. })\end{array}$ & $37 \%(22)$ & $24 \%(29)$ \\
$\begin{array}{c}\text { Raised alkaline } \\
\text { phosphatase } \\
(>100 \text { i.u.) }\end{array}$ & $37 \%(30)$ & $24 \%(42)$ \\
$\begin{array}{c}\text { Raised bilirubin } \\
(>0.8 \text { mg) }\end{array}$ & $40 \%(32)$ & $18 \%(34)$ \\
$\begin{array}{c}\text { Urinary bilirubin } \\
+ \text { ve } \\
\text { Markedly raised urea } \\
(>80 \text { mg) }\end{array}$ & $69 \%(38)$ & $25 \%(12)$ \\
$\begin{array}{c}\text { Raised neutrophil WBC } \\
(>8000)\end{array}$ & $73.7 \%(38)$ & $56.7 \%(60)$ \\
\hline
\end{tabular}

A group of thirty-two clinical cases of venous thrombo-embolism showed similar abnormal figures although abnormalities were less frequent. There was often an elevated blood urea in association with pulmonary embolism and the mean abnormal level was $147 \mathrm{mg} / 100 \mathrm{ml}$ with a control of $80 \mathrm{mg} / 100 \mathrm{ml}$.

No biochemical test could be considered as a screening or a specific test for pulmonary embolism. A rising blood urea or abnormalities of liver function were suggestive if other causes had been eliminated. The value of the triad of Wacker, Snodgras and Amador (1951), i.e. a raised serum LDH and bilirubin but a normal SGOT, was not confirmed. Clinical studies of Cugell et al. (1967), Wacker and Snodgras (1960) and Israel and Goldstein (1957) have given conflicting results, but autopsy studies with controls were lacking.

The elevation of the urea may be due to reduced cardiac output and the abnormality of liver function may be due to associated hepatic congestion and haemolysis in a pulmonary infarction (Rich and Resnick, 1962). A moderate leucocytosis is usual after pulmonary infarction and the ESR is raised, but these are not of diagnostic value (Miller and Berry, 1951).

\section{Electrocardiographic features}

The electrocardiograms of the patients included in the previous section (excluding those with evidence of acute myocardial infarction) were analysed. Abnormalities of the QRS, the T wave and the rhythm were recorded separately and the classical abnormality of pulmonary embolism was considered to be an S1Q3R3 with T wave inversion in III ( \pm avf) and V1 to V4 (Wood, 1941) (Fig. 1).

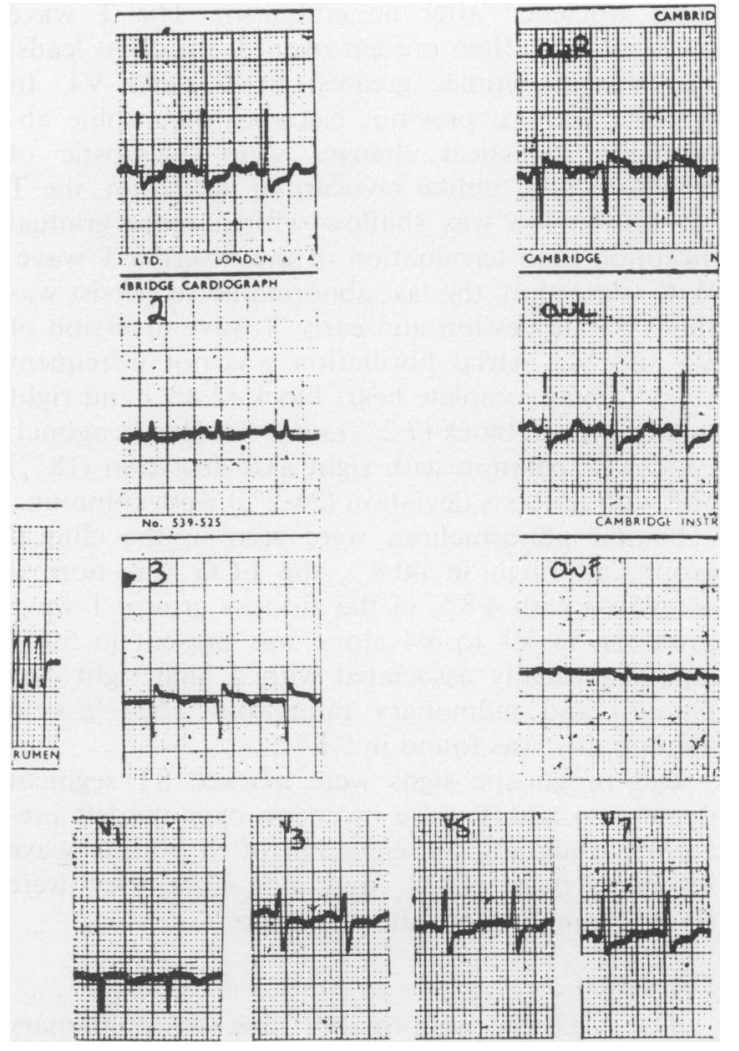

Fig. 1. Electrocardiogram of acute cor pulmonale due to pulmonary embolism.

\section{Results}

There were eighty-three cases with electrocardiograms and evidence of emboli at autopsy. No abnormality was found in the embolic group alone. Sixteen autopsy cases with emboli had the classical change but this was also present in ten of the control group. There were no cases of myocardial infarction secondary to pulmonary embolism, although four cases showed ECG evidence of inferior myocardial infarction.

$\mathrm{T}$ wave inversion confined to V1-V4 without right axis deviation regressing over the next 2 weeks was found predominantly in patients with right lower lobe infarction or infection. There were six autopsy cases with pulmonary atheroma and right ventricular hypertrophy thought to be due to pulmonary embolism/thrombosis. There were secondary $\mathrm{T}$ wave changes in V1-V4 but the ECG abnormalities were the same as three cases of idiopathic pulmonary hypertension seen during this period.

Serial ECGs were particularly valuable and often showed that ischaemic changes over leads 1,11 , V4-V6 $(15 \%)$ and occasionally over the inferior 
leads worsened after an embolism. The $\mathbf{T}$ wave inversion was often present over all the chest leads, and was sometimes greatest in V3 and V4. In patients without previous electrocardiographic abnormality, classical changes were diagnostic of embolism and, unlike myocardial infarction, the $\mathrm{T}$ wave inversion was shallow with a more gradual inception than termination of the inverted $T$ wave. With regression, the last abnormality to persist was slight ST depression and early $\mathrm{T}$ wave inversion of V1 and V2. Atrial fibrillation was not infrequent $(14.5 \%)$ and complete heart block $(2.4 \%)$ and right bundle branch block ( $7 \cdot 2 \%$ ) were not always agonal. Clockwise rotation with right axis deviation $(18 \%)$ and with left axis deviation (24.1\%) were common.

Similar abnormalities were seen in the clinical group, although in $40.8 \%$ the ECG was normal compared with $4.8 \%$ of the autopsy group. $T$ wave inversion in $\mathrm{V} 1$ to $\mathrm{V} 4$ alone was present in $5.1 \%$ and was usually associated with a high right diaphragm and pulmonary infarction. The classical abnormality was found in $9 \cdot 6 \%$.

Bad prognostic signs were marked ST segment depression and $T$ wave inversion over the left precordial leads and ST elevation often with $T$ wave inversion over 11,111, and avf, since they were found mainly in the autopsy group.

\section{Discussion}

The classical abnormality due to pulmonary embolism occurred in other conditions and only serial changes from or to normality were diagnostic. Other signs (McGinn and White, 1939; Duner, Pernow and Rigner, 1960) were of little value.

Caird and Stanfield (1962) found only marked clockwise rotation and a $P$ wave vector of $+70^{\circ}$, but rarely right precordial inverted $\mathrm{T}$ waves, in asphyxial cor pulmonale. These differences from thromboembolic cor pulmonale were not found in this study and the conditions often co-existed. A P pulmonale was rarely found with thrombo-embolism but the $T$ waves were usually asymmetrically inverted over the right ventricular leads, whereas in asphyxial cor pulmonale they were often symmetrically inverted. Right ventricular hypertrophy (Lipman and Massie, 1965) was rare except in thrombo-embolism.

Arrhythmias particularly atrial fibrillation (Short, 1952) and ventricular ectopics were common with emboli and both forms of cor pulmonale, possibly owing to associated ischaemic heart disease. The association of right lower lobe lesions with $T$ wave inversion in V1 to V4 only was probably due to the elevation of the diaphragm that was found.

\section{Conclusions}

Clinical features of pulmonary embolism without infarction are never diagnostic and in the elderly unexplained tachypnoea, stupor, hypotension and ankle oedema may be the only signs. It is often massive and unsuspected. It should be considered when the lungs are clear and the normal chest radiograph seems incompatible with the severity of the patient's condition. Rhonchi may be heard in patients with previous obstructive airways disease (Webster et al., 1966) but are otherwise uncommon. Impairment of liver function and a rising blood urea may be indicators in the elderly, since the urea is often low in primary hepatic disorders. In cases of sudden onset, particularly with right ventricular failure and shock, the electrocardiographic changes may be diagnostic but, without previous records, the lesser changes are of no diagnostic value.

The differential diagnosis of pulmonary infarction is predominantly pneumonia and neoplasia, since dyspnoea, haemoptysis and pleuritic pain are the usual features (Barritt and Jordan, 1961). Unilateral impairment of air entry and rales are common but the latter are not bilateral unless there is coincidental pulmonary oedema. Apart from the history, the chest radiograph is most useful in diagnosis. Widespread use of pulmonary angiography is impractical. However, the lung scan can be repeated frequently although it is not specific. Therefore, in doubtful cases an abnormal lung scan must be interpreted cautiously and if repetitive episodes occur with an abnormal lung scan, pulmonary angiography is essential. The absence of false-negatives makes scanning the best screening test and a discrete lobar or sublobar lack of uptake is highly suggestive of neoplasm or embolism.

\section{Acknowledgments}

We are grateful to the Physics Department and the Medical Records Department, Nottingham General Hospital, for their assistance, and to Dr C. S. Darke for his helpful advice.

\section{References}

BarritT, D.W. \& Jordan, S.C. (1961) Clinical features of pulmonary embolism. Lancet, i, 729.

Beerel, F.R., Vance, J.W., Cordasco, E.M., Wende, R.W. \& Toffolo, R.R. (1969) Angiograms, laminagrams and lung scans in emphysema. Archives of Internal Medicine, $124,8$.

CAird, F.T. \& Stanfield, C.A. (1962) The electrocardiogram in asphyxial and in embolic cor pulmonale. British Medical Journal, 3, 24.

Carlotti, J., Hardy, J.B., Linton, R.R. \& White, P.D. (1947) Pulmonary embolism in medical patients. Journal of the American Medical Association, 134, 1447.

Cugell, D.W., Buckingham, W.B., Webster, J.R. \& KeITEL, L.J. (1967) The limitations of laboratory methods in the diagnosis of pulmonary embolism. Medical Clinics of North America, 51, 175.

Duner, H., Pernow, B. \& Rigner, K.G. (1960) The ECG pattern in pulmonary embolism. Acta medica scandinavica, 168, 397.

Haynie, T.P., Hendrick, C.K \& Schreiber, M.H. (1965) Diagnosis of pulmonary embolism and infarction by photoscanning. Journal of Nuclear Medicine, 6, 613. 
IsRael, H.L. \& Goldstein, F. (1957) The varied clinical manifestations of pulmonary embolism. Annals of Internal Medicine, 47, 202.

JAmes, A. E., CoOper, M., White, R.I. \& WAgner, H.N. (1971) Perfusion changes on lung scanning in patients with congestive heart failure. Radiology, 100, 99.

Lipman, B.S. \& MAssie, E. (1965) Clinical Scaler Electrocardiography. Fifth edition. Year Book Medical Publishers.

Maclean, A., Shibata, H.R., Mclean, A.P., Skinner, G.B. \& Gutelius, J.R. (1967) Pulmonary embolism and bedside scanning. Candian Medical Association Journal, $97,991$.

McGinN, S. \& White, P.D. (1939) Acute cor pulmonale resulting from pulmonary embolism. Journal of the American Medical Association, 104, 1473.

Miller, R. \& BerRY, J.B. (1951) Pulmonary embolism, a frequently missed diagnosis. American Journal of Medical Science, 222, 197.

Moser, K.M., Tisi, G.M., Rhodes, P.G., Landis, G.A. \& Miale, A.A. (1966) Pulmonary angiographic and radioscan correlation in patients with pulmonary embolism. Clinical Research, 14, 105.

Rich, A.R. \& ResNick, W.H. (1962) On the mechanism of jaundice following pulmonary infarction in patients with heart failure. Bulletin of the Johns Hopkins Hospital, 38, 75.

SHORT, D.S. (1952) A survey of pulmonary embolism in a general hospital. British Medical Journal, 1, 790.

WACKer, W.E.C. \& SNOdgras, P.J. (1960) Serum L.D.H. activity in the diagnosis of pulmonary embolism. Journal of the American Medical Association, 174, 2142.

W ACKer, W.E.C., Snodgras, P.J., Rosenthal, M. \& AMADOR, E. (1961) A triad for the diagnosis of pulmonary embolism and infarction. Journal of the American Medical Association, 178, 8.

WAGNER, H.N. (1968) Regional distribution of pulmonary arterial blood flow in acute asthma. Journal of the American Medical Association, 102, 267.

Wagner, H.N., Tow, D.E., Lopez-Majano, V., Chernick, V. \& Twining, R. (1966) Factors influencing regional pulmonary blood flow in man. Scandinavian Journal of Respiratory Diseases, Suppl. 62, 59.

Webster, J.R., SAadeh, G.B., Eggum, P.R. \& Sucker, J.R. (1966) Wheezing due to pulmonary embolism. New England Journal of Medicine, 274, 931.

Wood, P. (1941) Pulmonary embolism, diagnosis by chest lead electrocardiography. British Medical Journal, 3, 21. 